\title{
Free Space Laser Communications
}

\author{
Dr. James Lesh \\ Jet Propulsion Laboratory \\ California Institute of Technology
}

\section{Outline of Presentation}

- Fundamentals

- Spacecraft Technology

- Ground Reception Systems

- Simplified Link Calculation

- Recent Demonstrations

- Future Demonstrations 


\section{Fundamentals \\ Free Space Propagation}

- Electromagnetic beams diverge at rates at least as fast as $\lambda / \mathrm{d}$ (Diffraction-limit)

$-\lambda$ is the wavelength of the radiation

- $d$ is the diameter of the transmitting aperture

- $\mathrm{RF}$ wavelengths usually in the $\mathrm{cm}-\mathrm{m}$ range

- Optical wavelengths are in the $\mu \mathrm{m}$ range

- The more wavelengths across the aperture, the more narrow the beam divergence

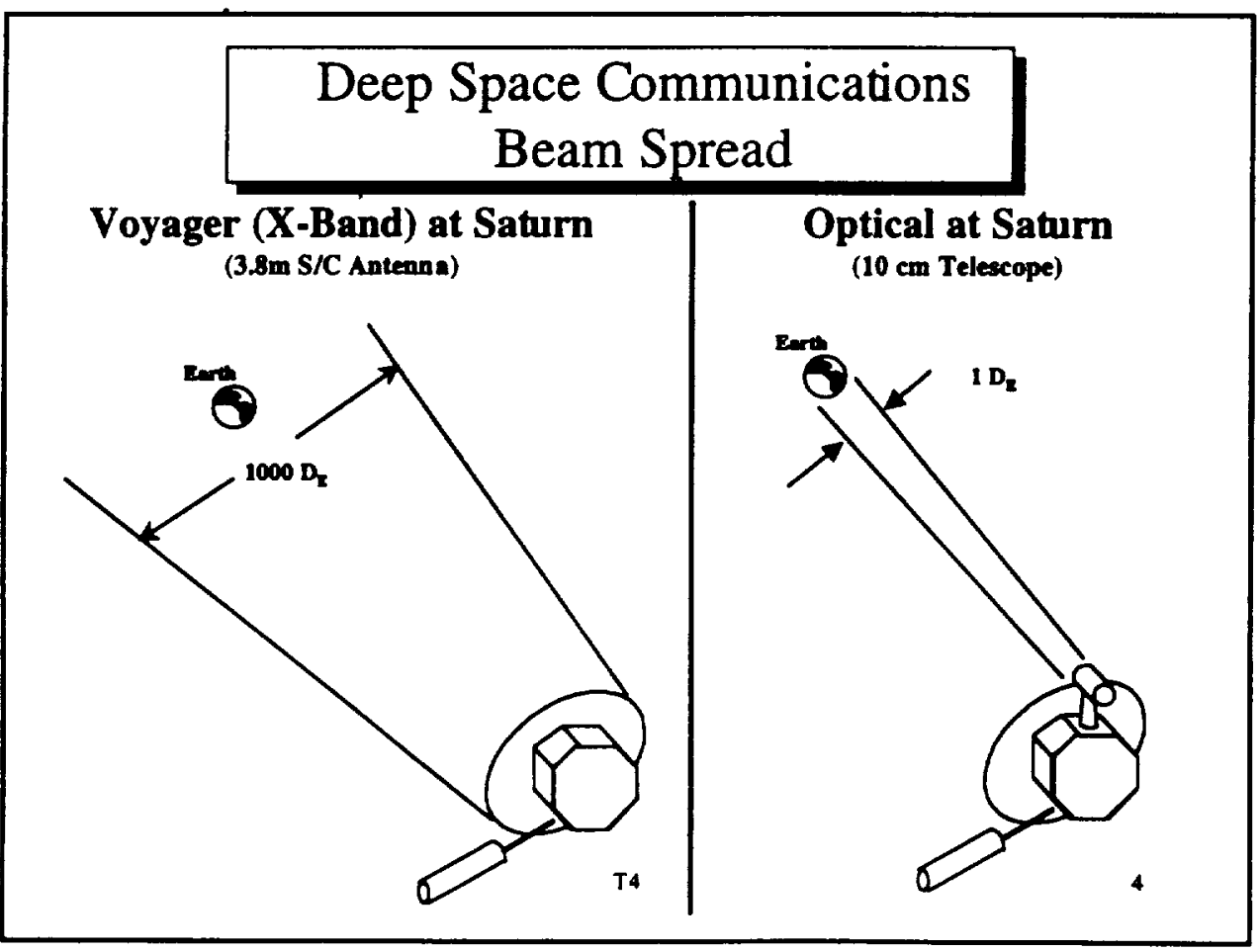



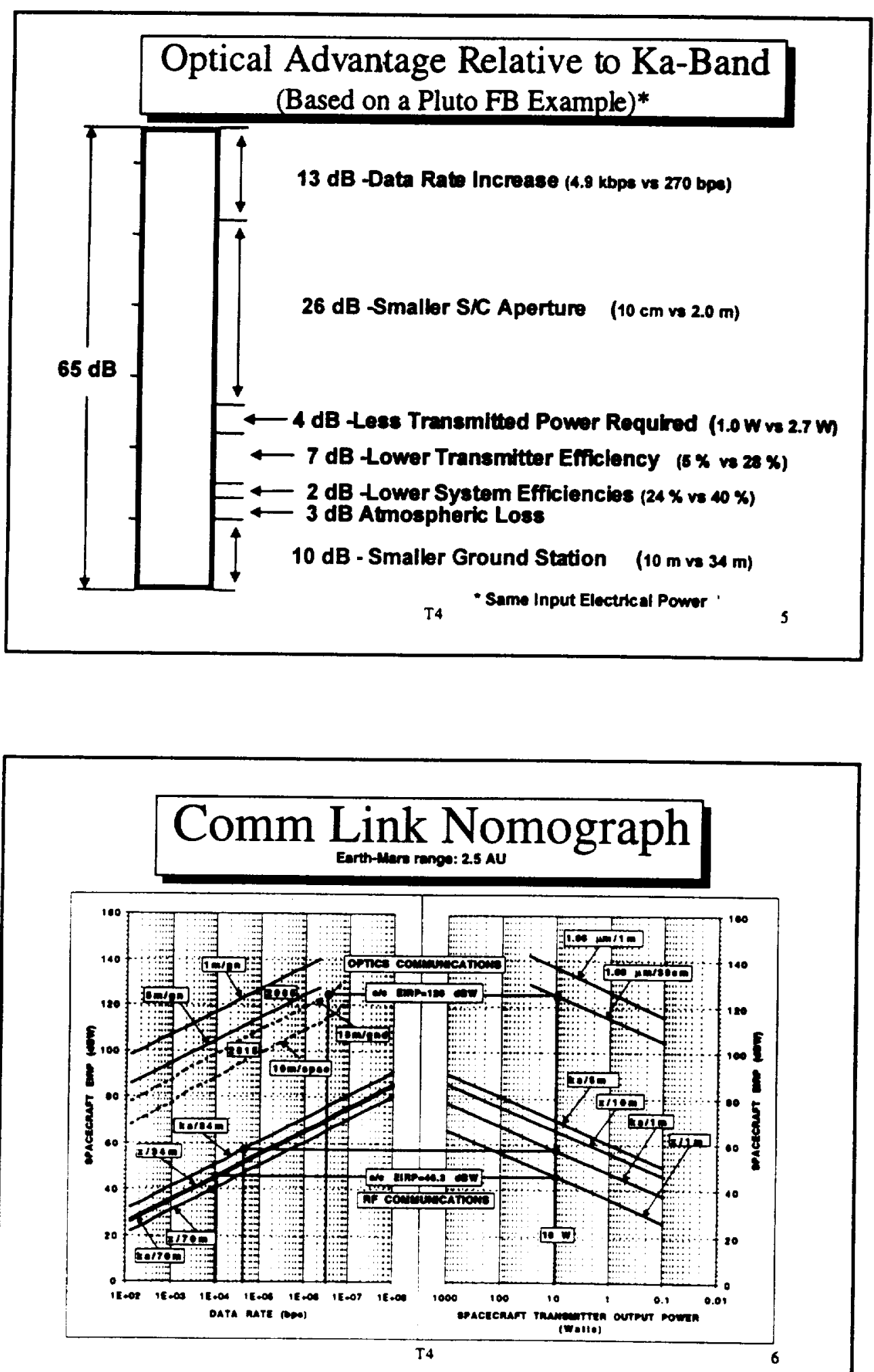


\section{Fundamentals}

\section{Equivalent $\mathrm{dB} / \mathrm{km}$ Loss for Free Space}

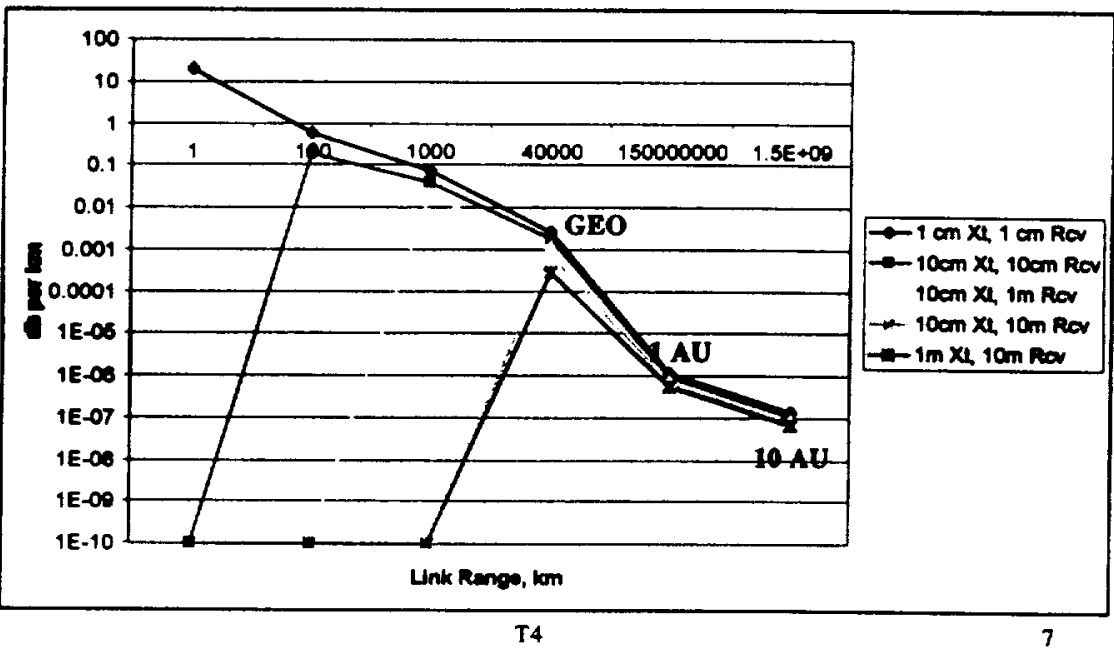

\section{Fundamentals}

Good News/Bad News

- Good News:

- Optical beams are more narrow

- Concentrate transmitted energy on target RCVR

- Bad News:

- Optical beams are more narrow

- Narrow beams must be more precisely pointed

- Must track beacon signal from intended receiver 


\section{Spacecraft Technology}

\section{Optical Communications Demonstrator (OCD) Simplified Optical Design}

- Uses only one steering mirror and one detector array for all beam control functions

- Eliminates many beam relay optics and need for large optical bench

- All optics are located on telescope body

- Fiber-coupled laser transmitter signal removes laser hear from optics

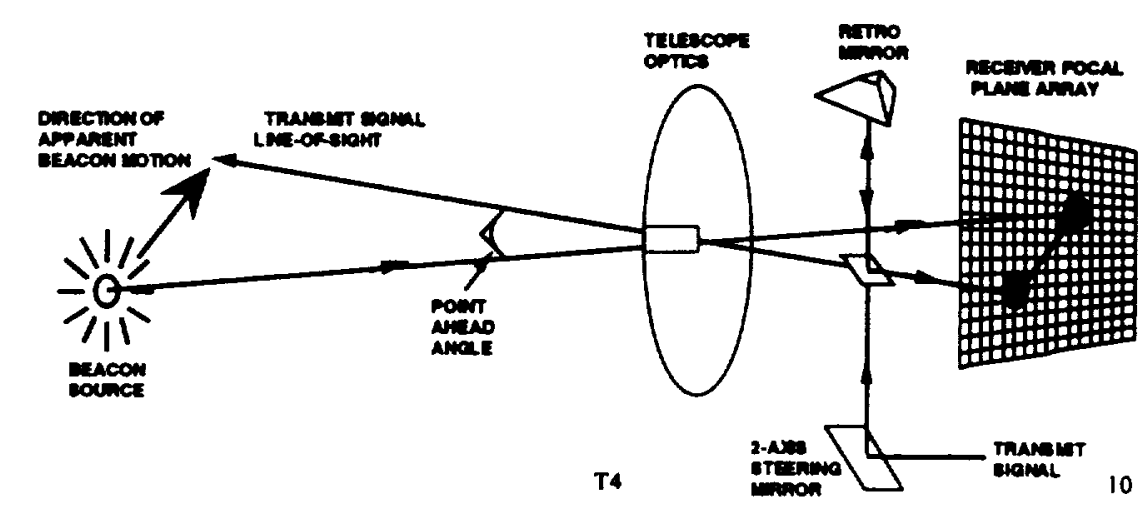




\section{Optical Communications Demonstrator}

Telescope Optical Assy (TOA)

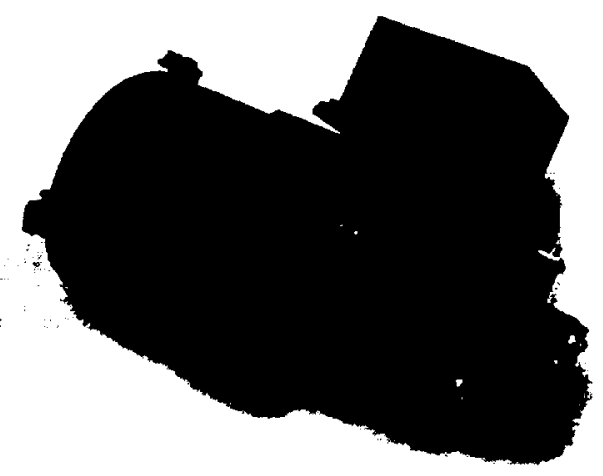

\section{Optical Communications Demonstrator} Terminal

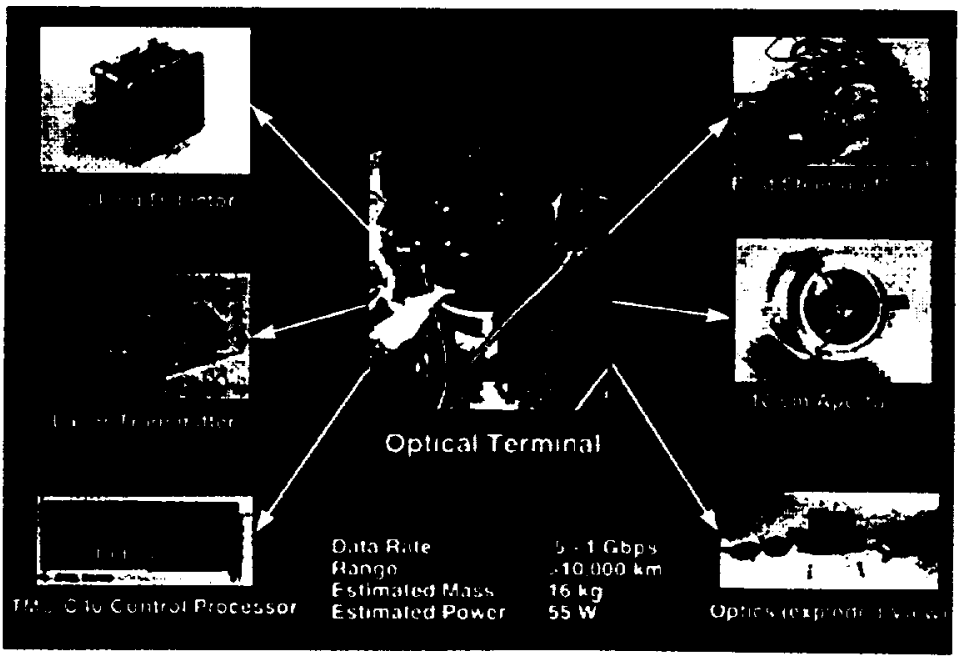




\section{OCD with Electronic Assy}

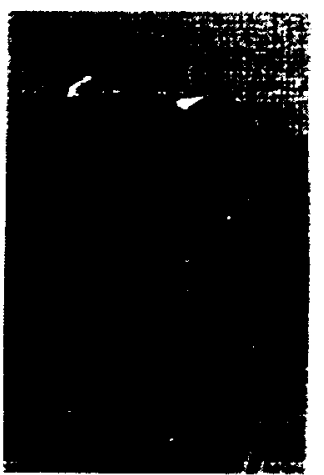

Telescope Optics Assembly (TOA) on gimbal

Control Electronics and Enclosure

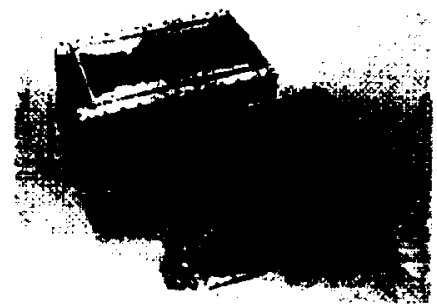

T4

\section{Optical Communications Demonstrator} Optics Layout

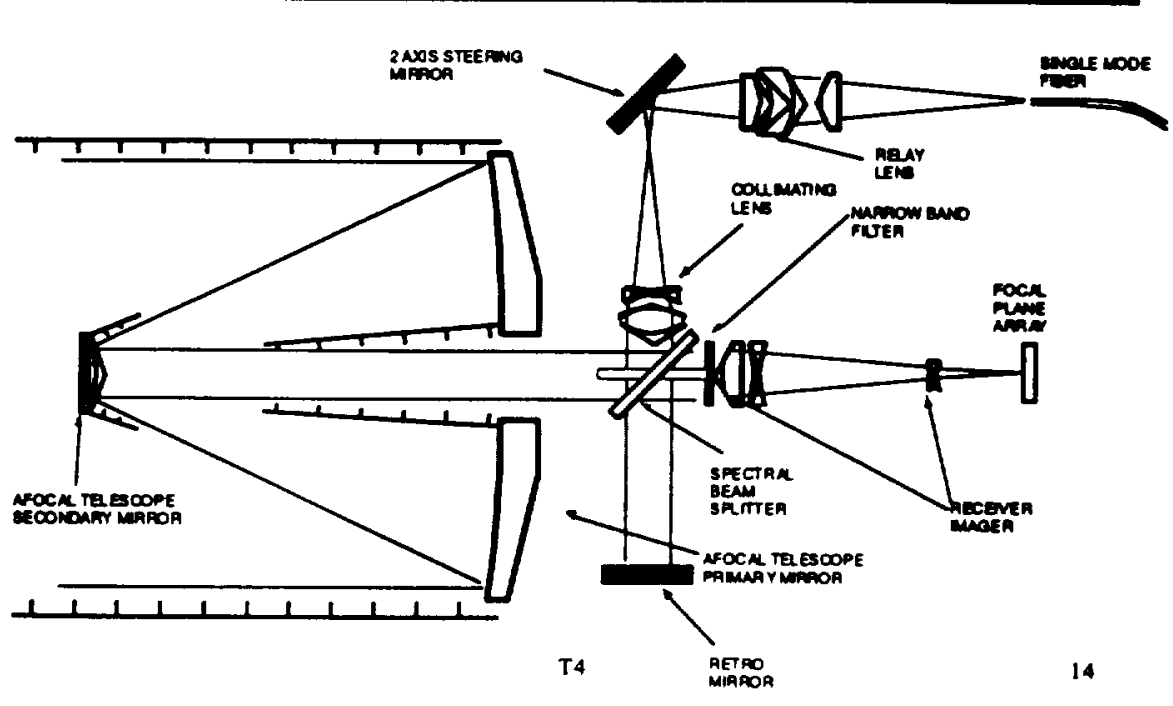




\section{(With Imager)}
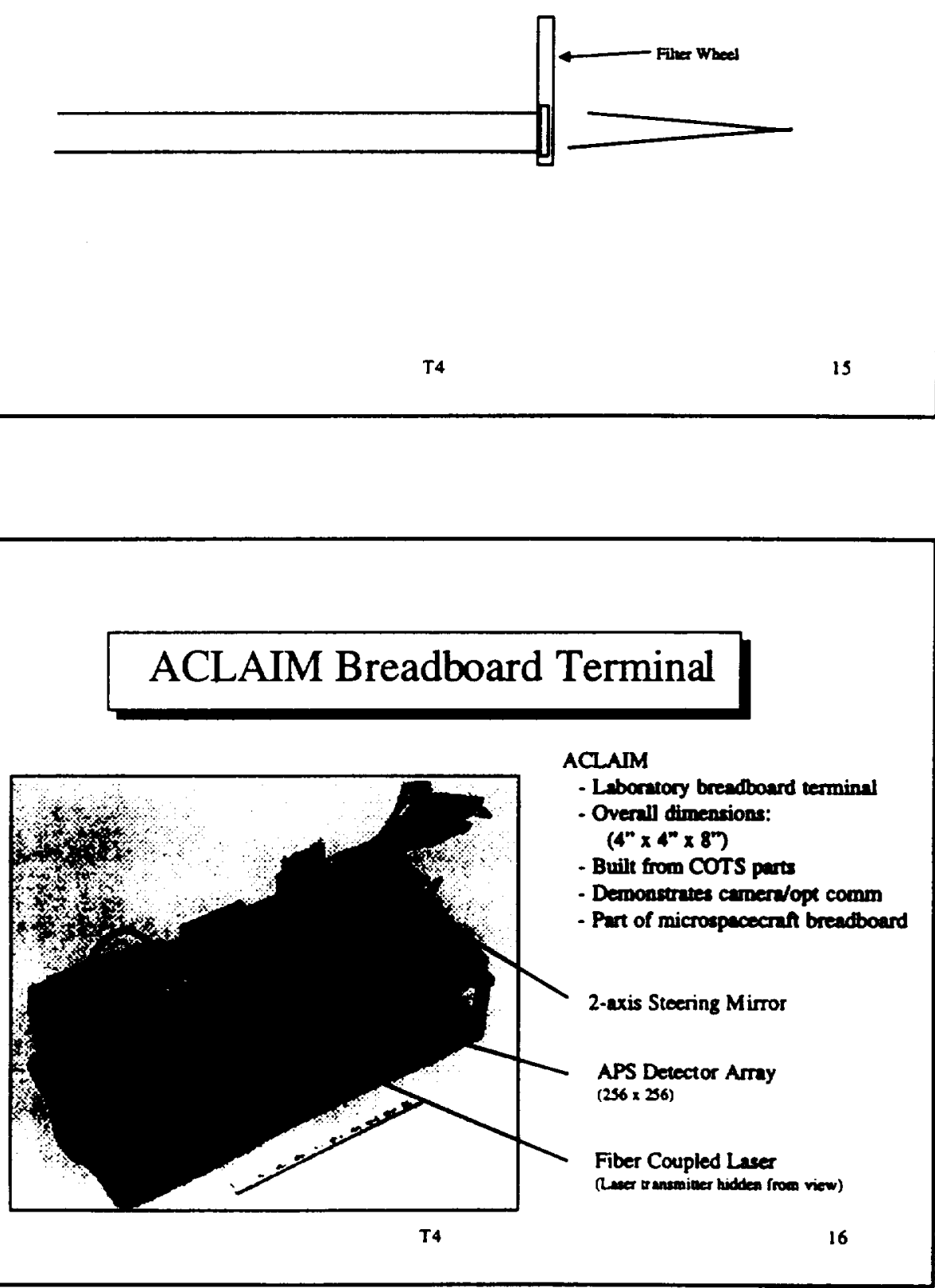


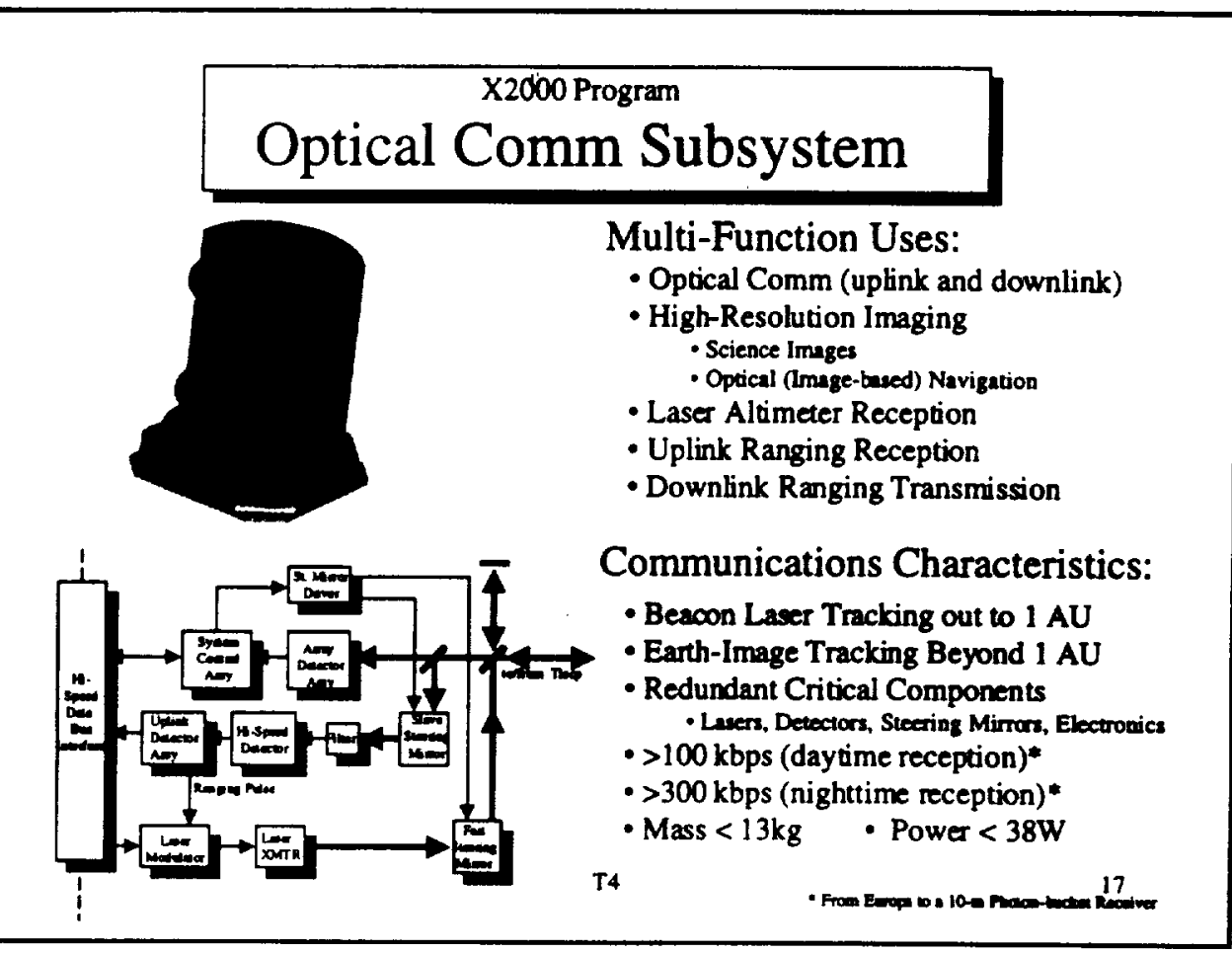

\section{JPL}

\section{2-WATT LASER DEVELOPMENT}

- DESIGNEv \& DEMONSTRATEDA MODLIATED, SOI.TD-STATE GKEEN LASER SOLRCE.

- GOAL. 2W OFGREENAT $50 \mathrm{KH}$. I'CLSE RATE

- ACHIEVED: 3.5 WATTS 11.7 WATTS CW AT INFRARED WAVELENCTH)

- LSES THRTE 10-WATT FIBER-COUPLED DIODE-LASER-BARS AS PUMP

- several cummercial companies INTERESTED IN DESICN
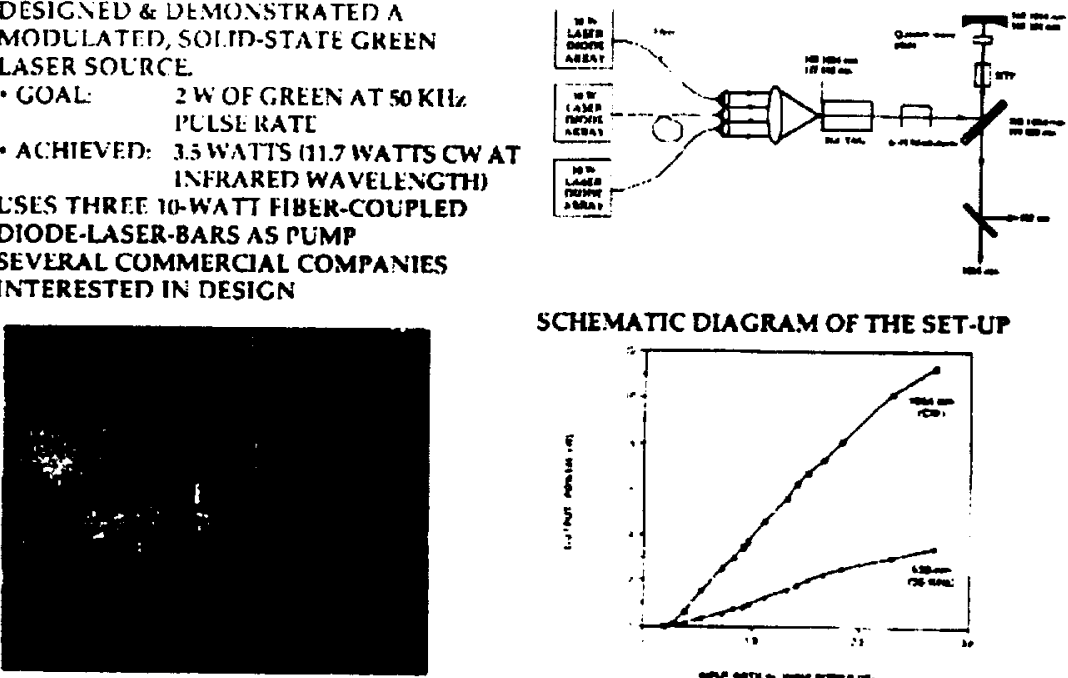

PICTURE OF TIIE SET.LP
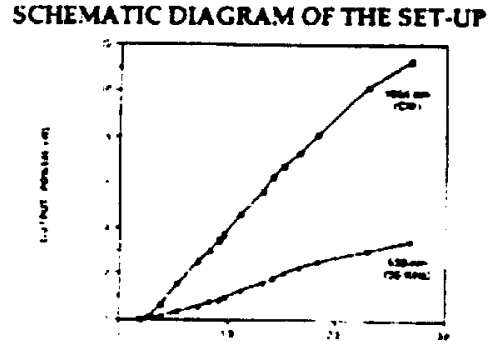

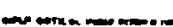

OLTPLT HOWEK WS IVPUT POWER 


\section{Lasercom Test and Eval Station}

- LTES is a high optical qually Instrument that characterizes the performance of iner communications tominab (LCT's)

- Mesures beam dhergence, acquibltion and tracking performance, optical output power, and BERs of LCTs up to 1.4 Gbpe data rates

- Appropriate exchange of beameplitters and detectors allowe spectral operating range to extend from 0.5 um ro $2 \mu \mathrm{m}$
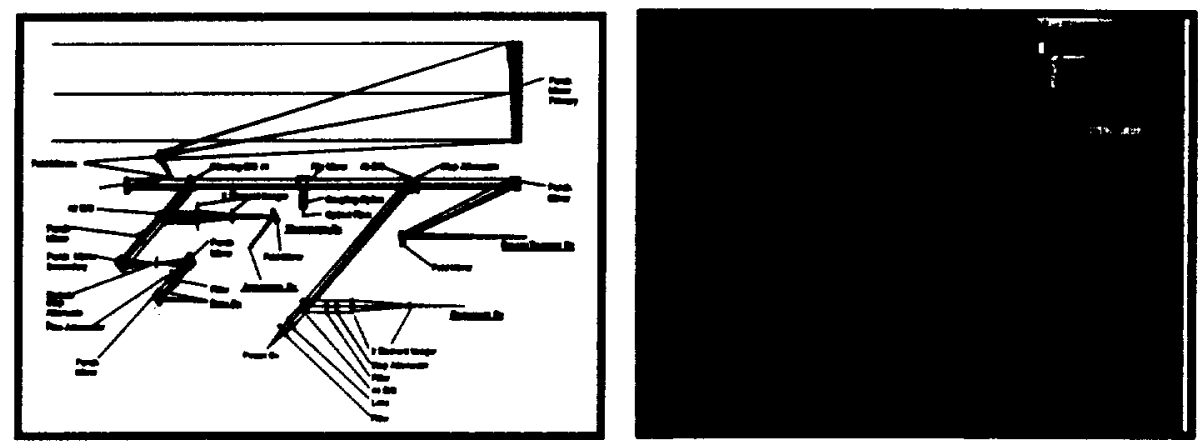

\section{Ground Reception Systems}




\section{1-m Optical Comm R+D Facility}

- Optical Comm Telescope Laboratory (OCTL)

- Located at JPL's Table Mountain Facility

- $2.4 \mathrm{~km}(7400 \mathrm{ft})$ elevation

- 1-m diameter aperture

- Fast (Earth-orbit) tracking mount

- Completion at end of 2000
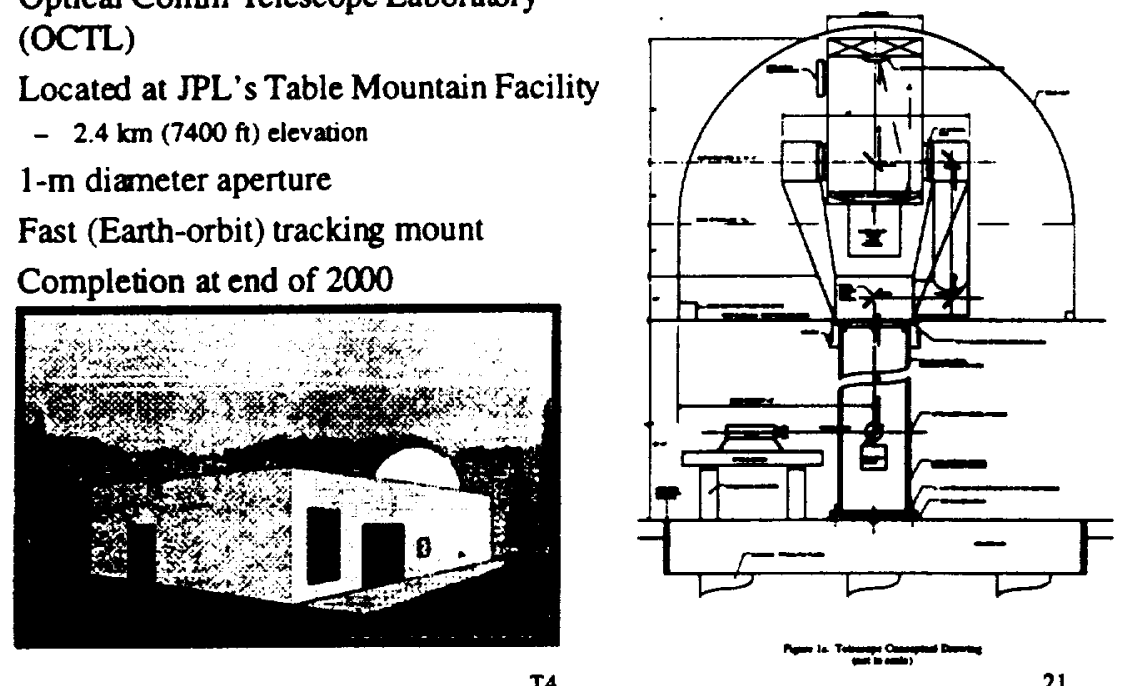

\section{Atmospheric Transmission}

\section{Clear Weather}

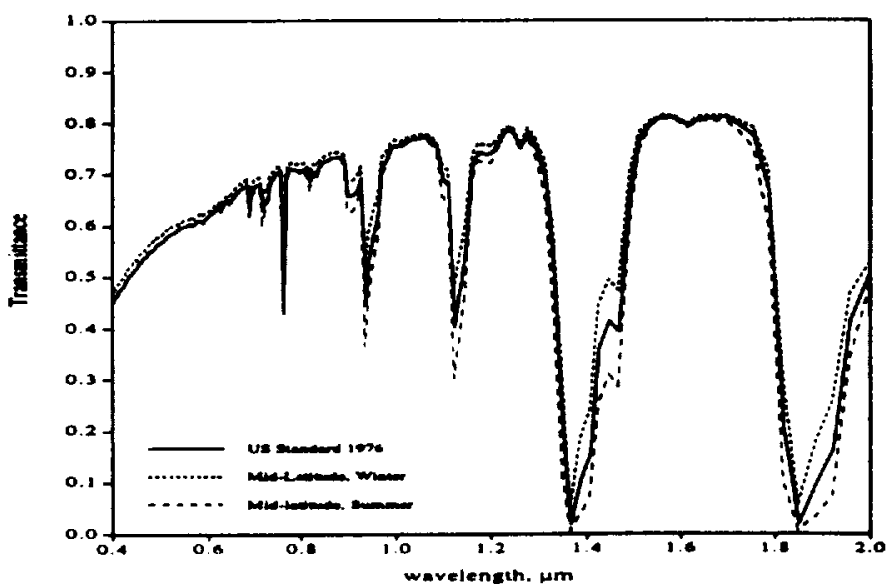




\section{Atmospheric Visibility Data}

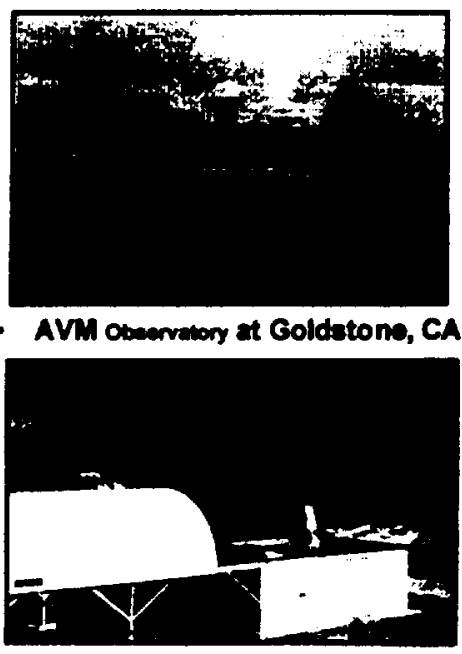

- Vislbility Cumulative Distribution

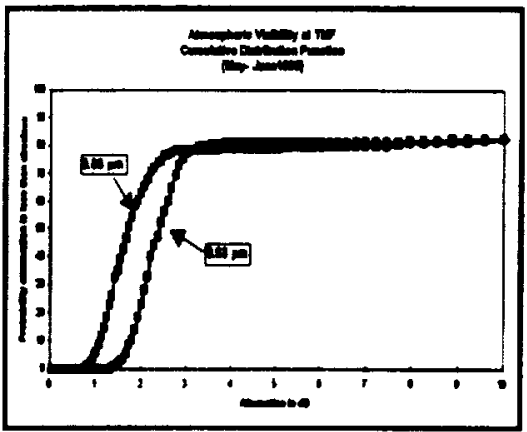

- AVM Obeervatory at Table Mtn, CA

\section{Deep Space Reception Station}
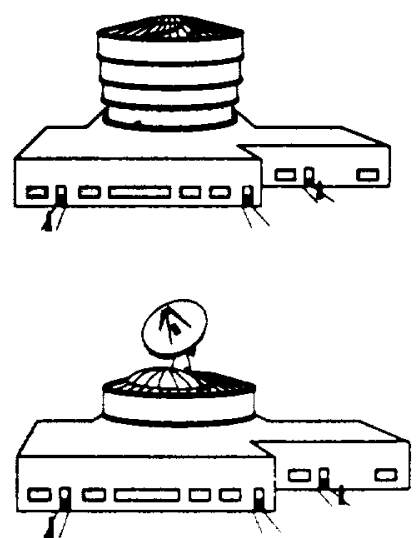

- 10-m collection aperture

- Photon bucket (non-diffractionlimited)

- Segmented primary mirror

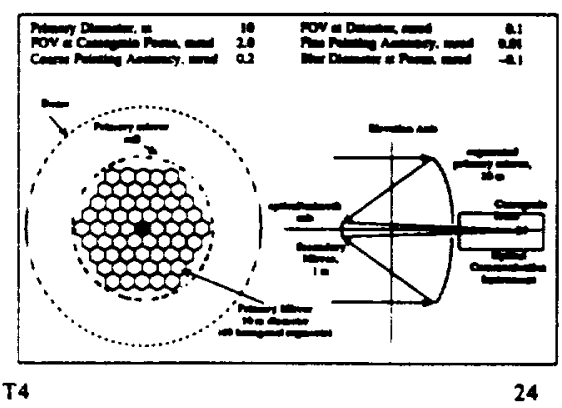




\section{Simplified Link Calculation}

\section{Simplified Link Calculation}

(Signal Level at Reciver)

- Calculate transmit beam divergence, $\theta=\lambda \mathrm{d}$

- Calculate spot diameter, $Z$, at target $R$ meters away using $Z=R * \theta$

- Calculate area of illuminated spot $\left(\pi Z^{2} / 4\right)$

- Area of receiver $=\pi D^{2} / 4$ (D-receiver diameter)

- Propagation loss $\left(L_{2}\right)$ is fraction of signal intercepted (receiver area) relative to total spot area $=D^{2} / Z^{2}$

- Received power $P_{r}$ (Watts) $=P_{1}^{*} L_{2} * T_{2}^{*} T_{w 0} * T_{n o}$

- $P_{1}=$ Transmitted power $\quad-T_{2}=$ Atmospheric Transmission

- $T_{w}=$ Transmit Optics Thruput $-T_{r o}=$ Receive Optics Thruput

- Received signal rate $=\mathrm{Pr} /(\mathrm{hv}$ ) (photons/sec)

$$
h v=\frac{2012}{\lambda(\text { in micions) }}
$$




\section{Simplified Link Calculation}

(Background Level at Receiver)

- Background Effects

- Point source interference signals produce a background flux rate over the receive aperture and over a spectral bandwidth (Watts/ $\mathrm{m}^{2 *} \mathrm{~nm}$ ) if in the detector field-of-view

- Distributed sources (e.g. daylight) provide a background flux rate over the receive aperture over the entire field-of-view of the receiver (Watts/ $\mathrm{m}^{2 *} \mathrm{~nm} \mathrm{~m}^{*} \mathrm{Sr}$ )

- Background signals are limited by narrow band filters of BW (in nm) and by detector FPV (in Sr)

- Received background power $\left(P_{b}\right)=$ background flux leved*Receiver area* filter BW ( ${ }^{*}$ FOV if extended source)

- Background Noise rate $=P_{b} /(h v)$ (in photons $/ \mathrm{sec}$ )

\section{Simplified Link Calculation}

(Detection Performance)

- Signal Detection (performance depends on type of detector, coding, and background levels)

\begin{tabular}{|l|l|}
\hline \multicolumn{1}{|c|}{ Receiver Type } & \multicolumn{1}{c|}{ Sensitivity } \\
\hline Inexpensive Receiver & $>100$ photons/bit \\
\hline State-of-the-Art Receiver & $\sim 10-20$ photons/bit \\
\hline Low Background/Low Rate Rcvr & $<1$ photons/bit \\
\hline
\end{tabular}




\section{Comparison of Optical and RF Links}

- Optical links are often compared to RF links

- Need to use a common comparison basis

- But, optical and RF have some fundamental differences

- Weather affects RF and optical systems differently

- RF links experience weather fades infrequently

- Optical must consider spatial diversity reception from the start.

- Need to develop an optical link design methodology that enables comparison with RF but allows for uniqueness of the two technologies

\section{Optical Weather Statistics}

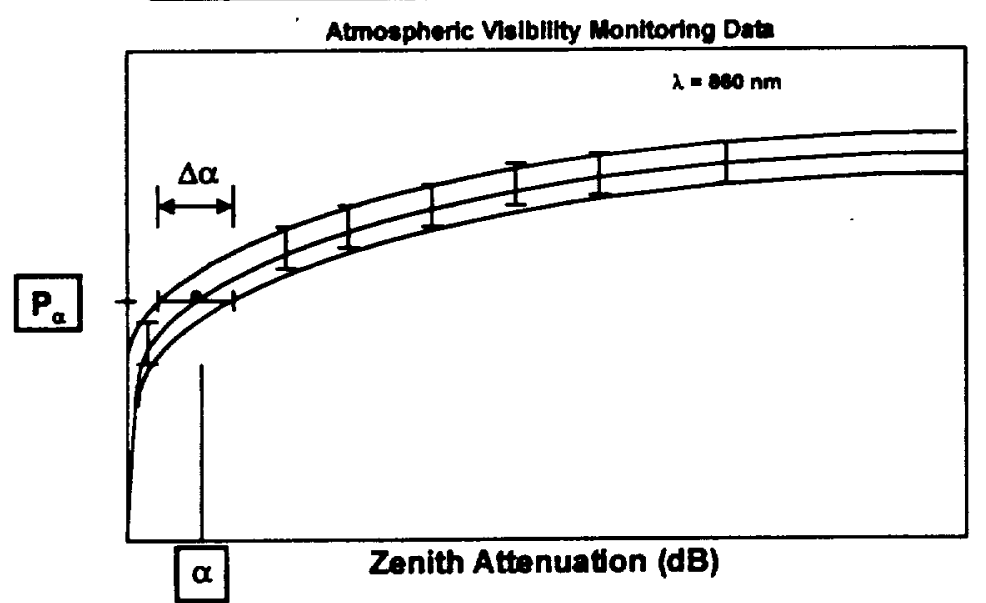

$\alpha$ - atmoaphark attonuation; $\Delta \alpha=$ attenuation uncertalnty; $P \alpha=$ prob(attenumition < a) Note: $\alpha$ must be adjusted tor operatlonal wavelength besed on known (LOWTRN) modols (if difierent from meseured wavelengths), and for olevation angle 


\section{Optical Weather Model}

- Atmospheric attenuation $(\alpha)$ is a continuous distribution ranging from low values (clear conditions) to very high vahues (due to clouds)

- Cloud outages impact "Station Availability"

- Mitigated by staion diversity

- Need to define what "outage" means

- Recommendation

- Use AVM data to define atmospheric model

- Select a value of $\alpha$ and the corresponding value of $\left(P_{a}\right)$

- $\mathrm{P}_{\alpha}=$ Probability that attenuation $<\alpha$

- Must be corrected for wavelength and elevation angle

- Approximate the AVM distribution by two states

- $<\alpha$ means clear (but with some attenuation)

- $>\alpha$ means (totally) obscured by clouds

- $P_{\alpha}$ determines station availability; $\alpha$ is nominal link attenuation and $\Delta \alpha$ is weather attenuation uncrtainty (when available)

\section{Link Analysis Using Weather Model}

- Analyze link using $-\alpha(\mathrm{dB})$ for atmospheric transmission and $+/-\Delta \alpha / 2$ as the favorable and adverse tolerances

- Design link Initially for a "Link Summary" of $0 \mathrm{~dB}$ margin using nominal parameter values and calculate the favorable $\left(+\sigma_{1}\right)$ and adverse $\left(-\sigma_{2}\right)$ uncertainties

- Calculate "Recommended Link Margin" based on the adverse link uncertainty (i.e. margin $=2 \sigma_{2}$ )

- Redo link design with a nominal link margin equal to the "Recommended Link Margin"

- Uses visibility data as a basis for link loss and link loss uncertainty

- Provides a formal basis for establishing value of link margin 


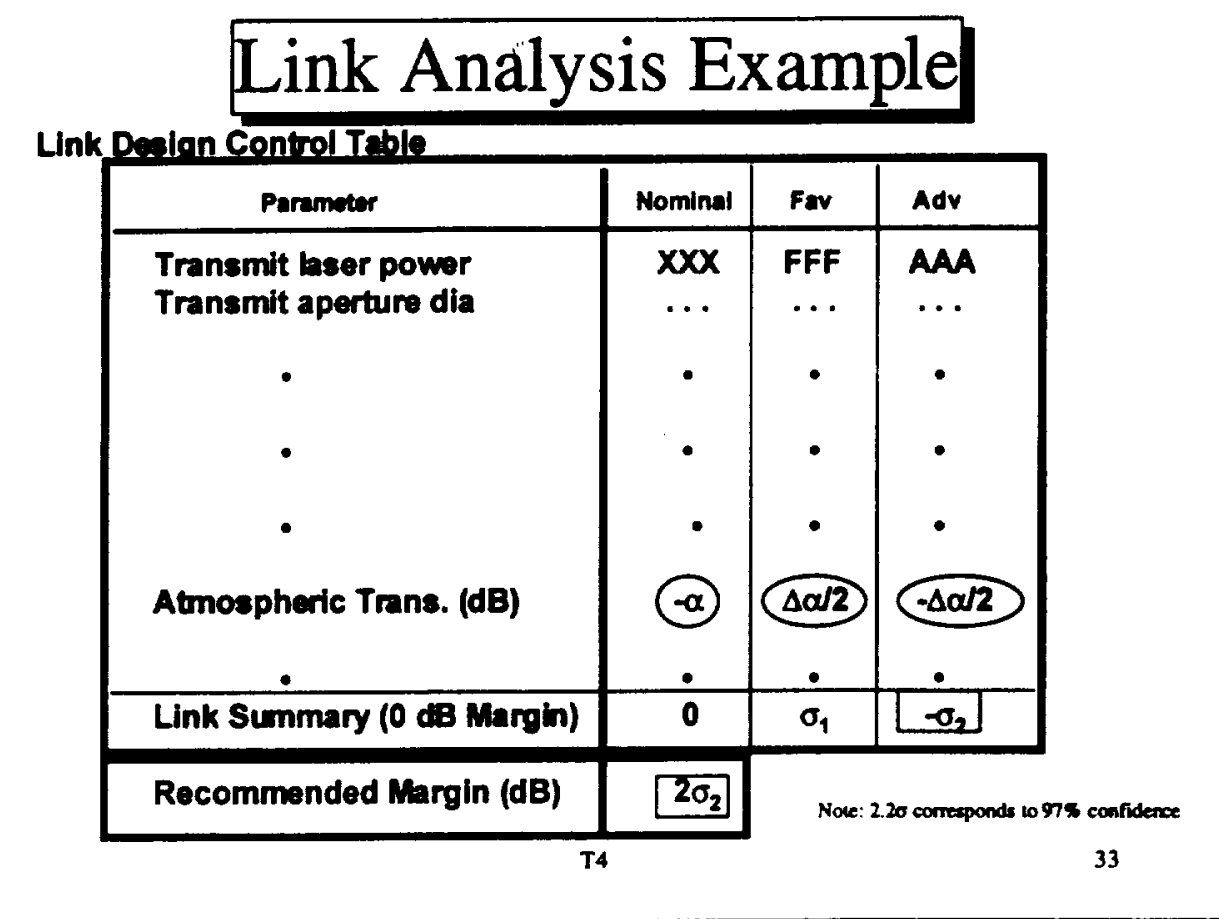

\section{Link Availability Analysis}

- Optical systems assume spatially-diverse reception

- Assume all ground stations are in independent weather cells (separated by few hundred $\mathrm{km}$ )

- Define a station as a "Candidate Station" if it can see spacecraft when atmosphere removed and above some minimum elevation angle (say 20 degrees)

- Define a station as "Available" if it is a candidate station and it has clear weather (i.e. atmospheric attenuation $<\alpha$ ) 


\section{Link Availability Analysis (Cont)}

If $\mathbf{N}$ stations are "Candidate Stations", then the probability that $m$ of them are "Available" is

$$
P_{N}(m)=\left(\begin{array}{l}
N \\
m
\end{array}\right)(P \alpha)^{m}(1-P \alpha)^{N-m}
$$

and the probability that at least one of the $\mathbf{N}$ stations is able to receive the link is

$$
P_{N}=\sum_{m=1}^{N} P_{N}(m)=1-\left(1-P_{\alpha}\right)^{N}
$$

\section{Link Availability Analysis (Cont)}

Next, consider total time (T) of spacecraft support "pass". Let $N_{1}$ be the number of candidate stations at the beginning of this time, and let the number of candidate stations change with time over the pass duration from $N_{1}$ (at the beginning) to $N_{K}$ at the end of the pass.

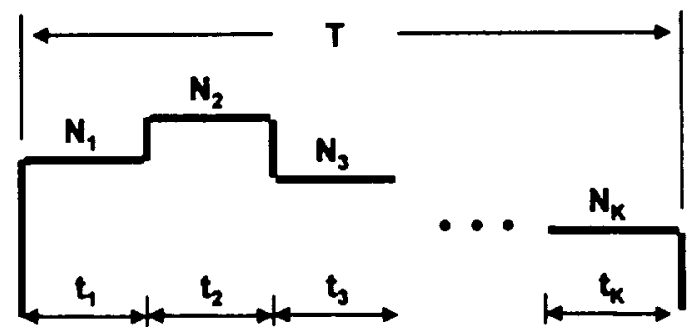

Let the corresponding times of $N_{1}$ candidate stations be $t_{1}$ 


\section{Link Availability Analysis (Cont)}

Then, the daily "Expected Data Volume" (EDV) returned for the link considered above, with the weather and station configuration being considered is

$$
E D V=R \sum_{i=1}^{k} t_{i} P_{N i}
$$

where " $R$ " is the data rate in the link design control table

RECOMMENDATION : Use EDV for RFIOptical comparisons

T4

\section{Recent Demonstrations}




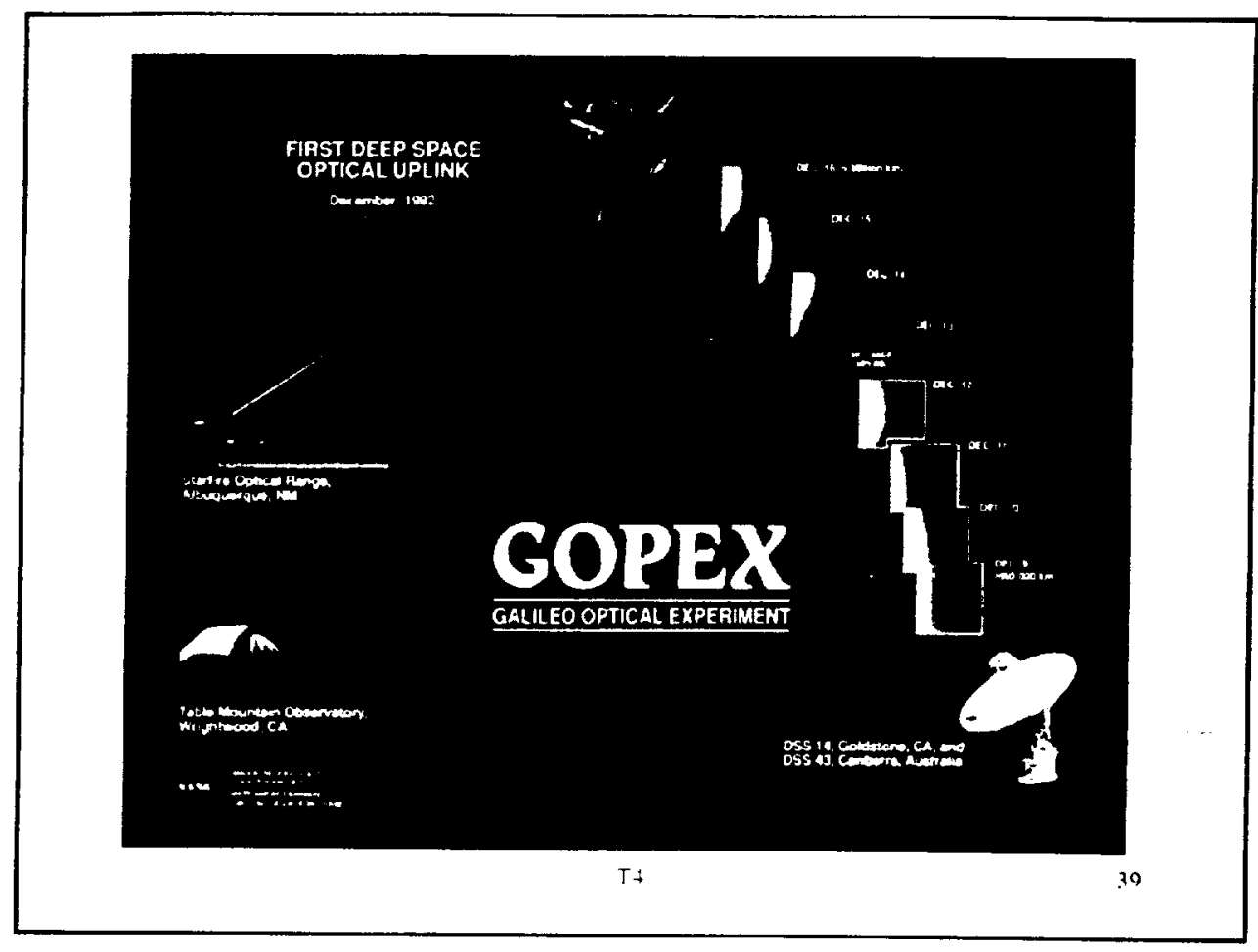

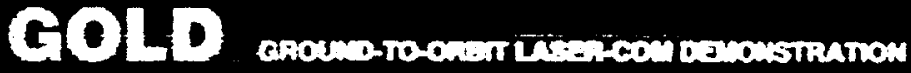

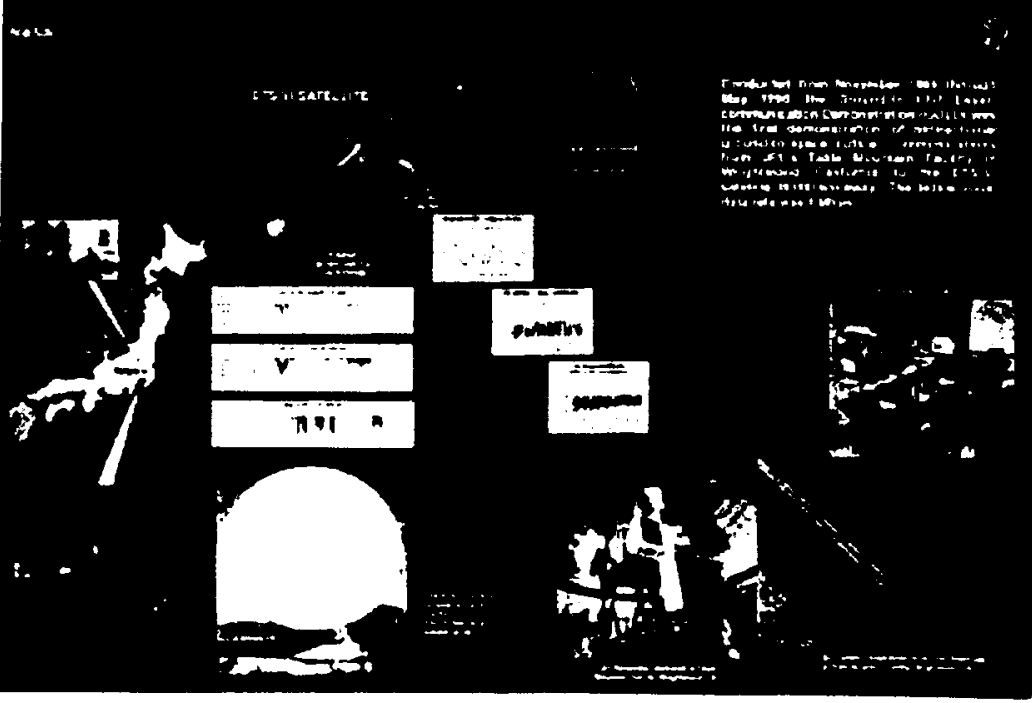




\section{Ground-Orbit Lasercom Demo (GOLD)}

GOLD Multiple-beam Transmission

- Multiple beam uplink mitigates effects of atmoepheric scintillation and beam wander

- Basms are propagated through difforent atmospherk coherent colls

- Each beam is delayed relative to the other by greater than lasers coherence lenoth
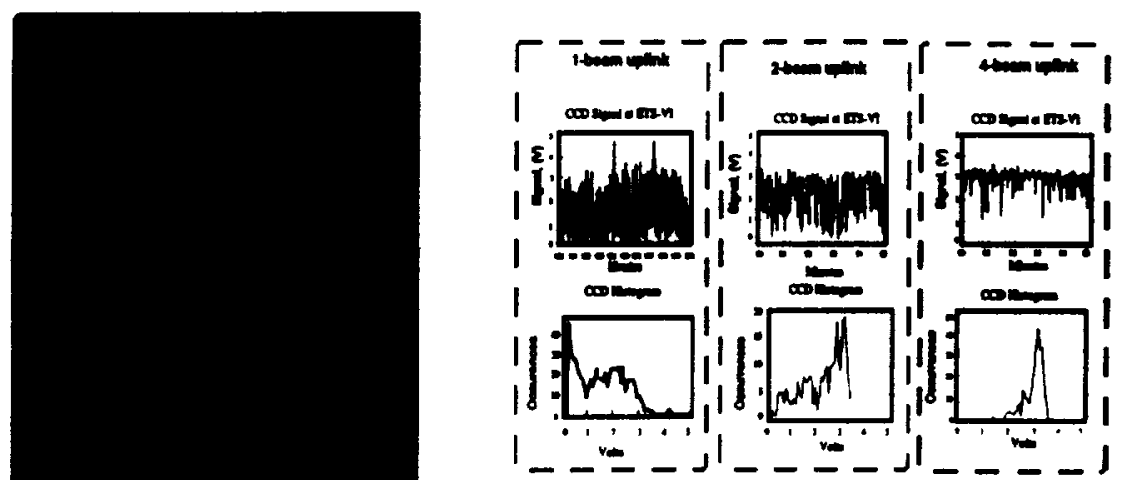

TMF 0.6-m Transmitler Telescope

\section{Future Demonstrations}




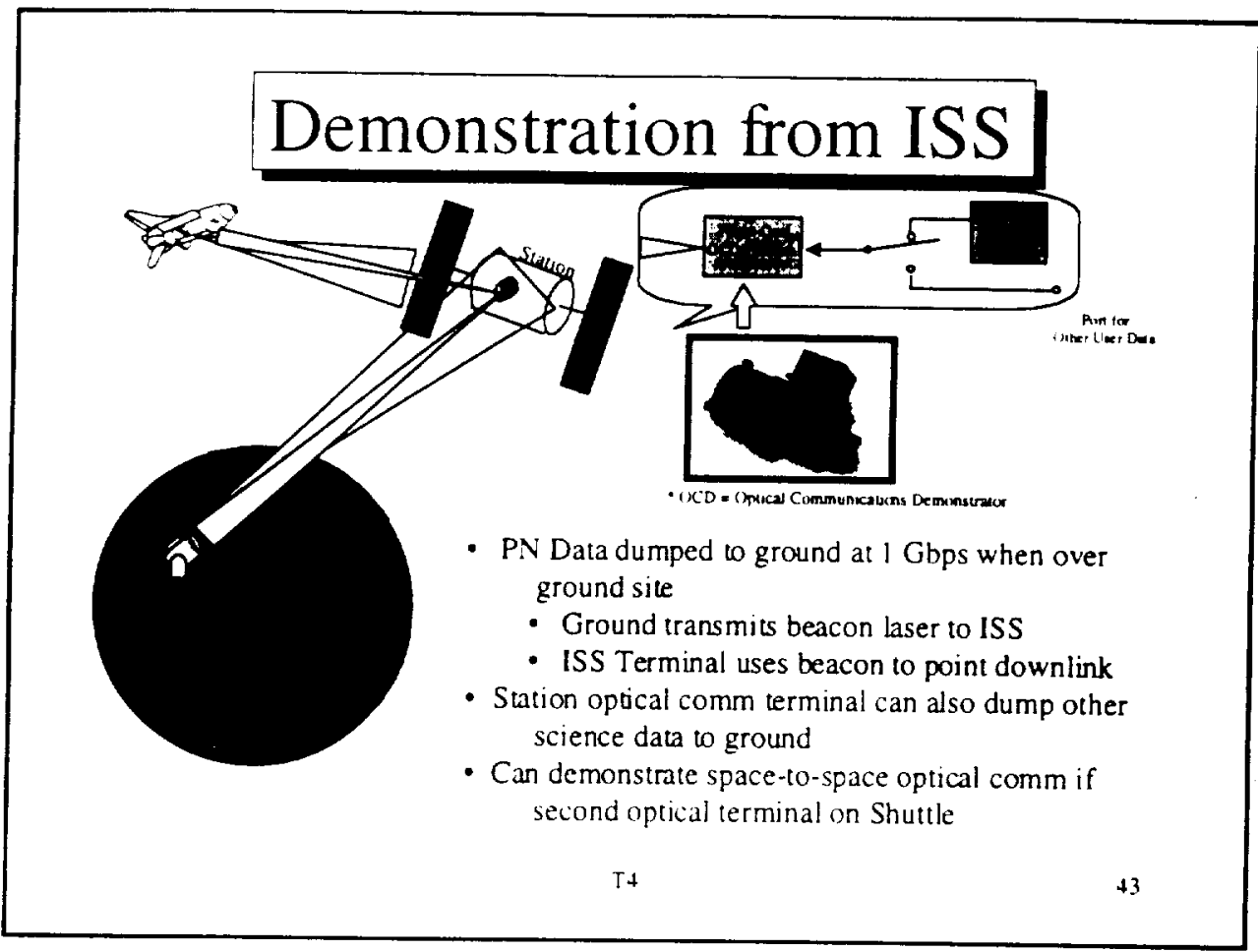

\section{Location of Flight Terminal}

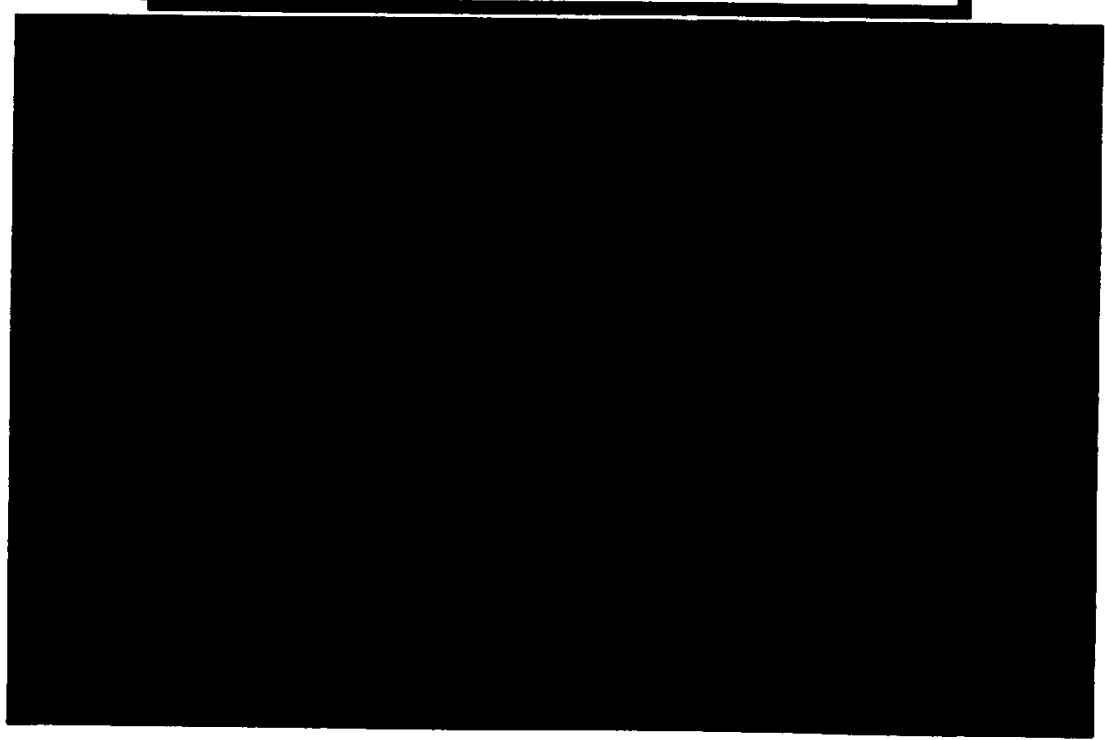




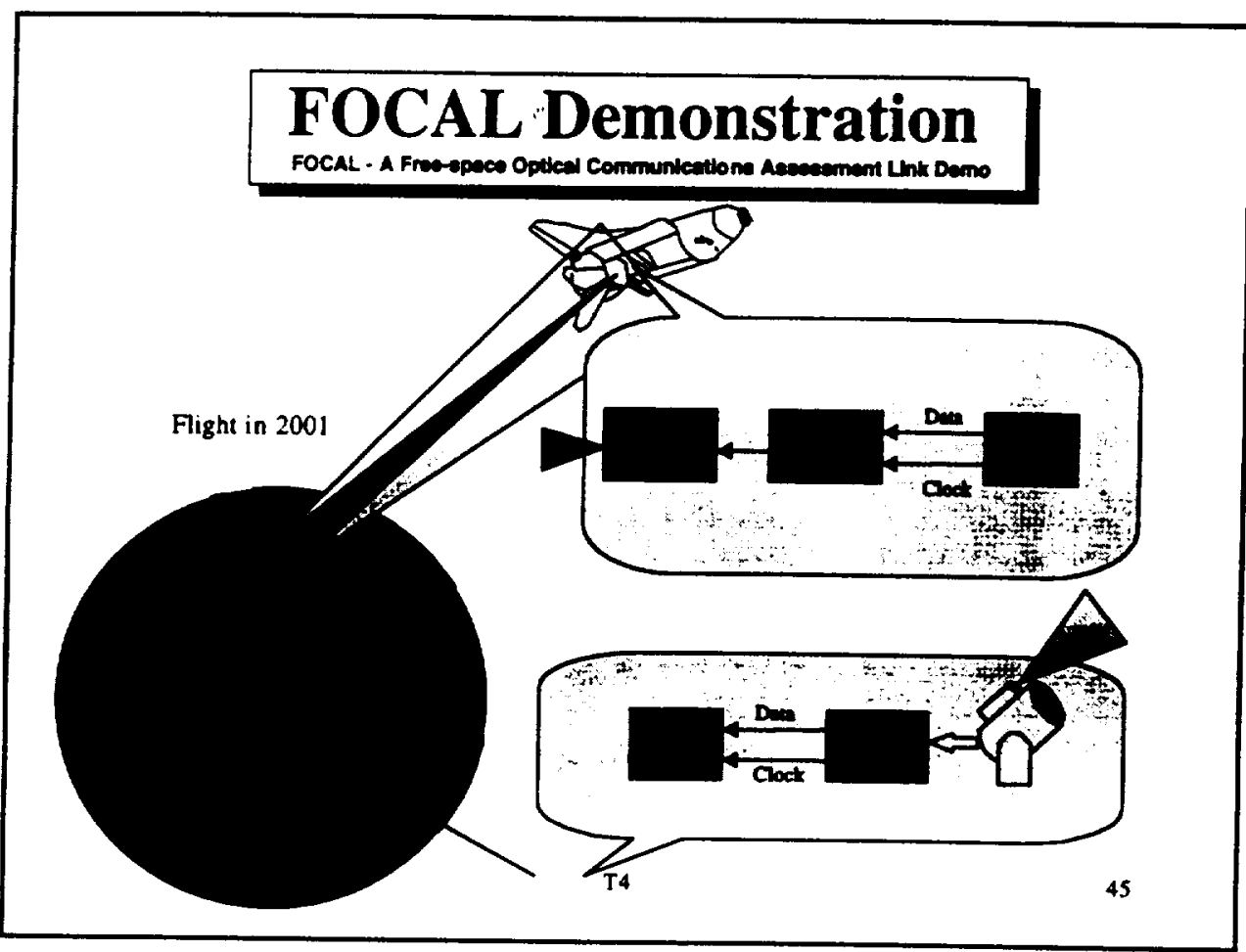

\section{Shuttle Link to Ground $1.6 \mathrm{Gbps}$}

Transmit Laser Power

Transmit Telescope Dia. (Space)

Link Range (Slant range)

Receive Telescope Dia. (Ground)

Atmospheric Losses (space-ground)

System Losses

Detector Efficiency

Data Rate

Link Margin

$\begin{array}{ll}100 & \mathrm{~mW} \\ 10 & \mathrm{~cm} \\ 1050 & \mathrm{~km} \\ 1 & \mathrm{~m} \\ 7 & \mathrm{~dB} \\ 5.2 & \mathrm{~dB} \\ 60 & \% \\ 1.6 & \mathrm{Gbps} \\ 21.3 & \mathrm{~dB}\end{array}$

